\title{
Galactic evolution of sulphur as traced by globular clusters ${ }^{\star \star \star}$
}

\author{
N. Kacharov ${ }^{1, \star \star \star}$, A. Koch ${ }^{1}$, E. Caffau ${ }^{2}$, and L. Sbordone ${ }^{3,4}$ \\ 1 Landessternwarte, Zentrum für Astronomie der Universität Heidelberg, Königstuhl 12, 69117 Heidelberg, Germany \\ e-mail: n.kacharov@lsw. uni-heidelberg.de \\ 2 GEPI, Observatoire de Paris, CNRS, Univ. Paris Diderot, Place Jules Janssen, 92195 Meudon, France \\ 3 Millenium Institute for Astrophysics, Av. Vicuña Mackenna 4860, 782-0436 Macul, Santiago, Chile \\ ${ }^{4}$ Pontifica Universidad Católica de Chile, Av. Vicuña Mackenna 4860, 782-0436 4860, Macul, Santiago, Chile
}

Received 26 September 2014 / Accepted 9 March 2015

\begin{abstract}
Context. Sulphur is an important volatile $\alpha$ element, but its role in the Galactic chemical evolution is still uncertain, and more observations constraining the sulphur abundance in stellar photospheres are required.

Aims. We derive the sulphur abundances in red giant branch (RGB) stars in three Galactic halo globular clusters (GC) that cover a wide metallicity range $(-2.3<[\mathrm{Fe} / \mathrm{H}]<-1.2)$ : M 4 (NGC 6121), M 22 (NGC 6656), and M 30 (NGC 7099). The halo field stars show a large scatter in the $[\mathrm{S} / \mathrm{Fe}]$ ratio in this metallicity span, which is inconsistent with canonical chemical evolution models. To date, very few measurements of [S/Fe] exist for stars in GCs, which are good tracers of the chemical enrichment of their environment. However, some light and $\alpha$ elements show star-to-star variations within individual GCs, and it is as yet unclear whether the $\alpha$ element sulphur also varies between GC stars.

Methods. We used the infrared spectrograph CRIRES to obtain high-resolution $(R \sim 50000)$, high signal-to-noise $(S / N \sim 200 \mathrm{per}$ px $)$ spectra in the region of the S I multiplet 3 at $1045 \mathrm{~nm}$ for $15 \mathrm{GC}$ stars selected from the literature (six stars in M 4, six stars in M 22, and three stars in M 30). Multiplet 3 is better suited for S abundance derivation than the more commonly used lines of multiplet 1 at $920 \mathrm{~nm}$, since its lines are not blended by telluric absorption or other stellar features at low metallicity.

Results. We used spectral synthesis to derive the [S/Fe] ratio of the stars assuming local thermodynamic equilibrium (LTE). We find mean $[\mathrm{S} / \mathrm{Fe}]_{\mathrm{LTE}}=0.58 \pm 0.01 \pm 0.20 \mathrm{dex}$ (statistical and systematic error) for $\mathrm{M} 4,[\mathrm{~S} / \mathrm{Fe}]_{\mathrm{LTE}}=0.57 \pm 0.01 \pm 0.19 \mathrm{dex}$ for M 22 , and $[\mathrm{S} / \mathrm{Fe}]_{\mathrm{LTE}}=0.55 \pm 0.02 \pm 0.16$ dex for M 30. The negative NLTE corrections are estimated to be in the order of the systematic uncertainties. We do not detect star-to-star variations of the $S$ abundance in any of the observed GCs, with the possible exception of two individual stars, one in M 22 and one in M 30, which appear to be highly enriched in S.

Conclusions. With the tentative exception of two stars with measured high S abundances, we conclude that sulphur behaves like a typical $\alpha$ element in the studied Galactic GCs, showing enhanced abundances with respect to the solar value at metallicities below $[\mathrm{Fe} / \mathrm{H}]-1.0$ dex without a considerable spread.
\end{abstract}

Key words. stars: abundances - Galaxy: halo - globular clusters: individual: M 4 - globular clusters: individual: M 22 globular clusters: individual: M 30 - globular clusters: general

\section{Introduction}

Sulphur is one of the less studied $\alpha$-elements (produced by sequential capturing of He nuclei) in stars. In general, the production site of the $\alpha$-elements such as $\mathrm{O}, \mathrm{Ne}, \mathrm{Mg}, \mathrm{Si}, \mathrm{S}, \mathrm{Ar}, \mathrm{Ca}$, and $\mathrm{Ti}$ is mainly associated with the eruptions of type II supernovae (SNe II). In contrast, the main fraction of the iron-peak elements abundance comes from $\mathrm{SNe}$ Ia, which contribute little or no $\alpha$-elements. The different timescales of the occurrence of SNe Ia or II makes the $[\alpha / \mathrm{Fe}]$ ratio a powerful tool for diagnosing the chemical evolution and star formation history (SFH) of any stellar population (Tinsley 1979; Matteucci \& Brocato 1990; McWilliam et al. 2013). In the Milky Way, the metal-poor stars $([\mathrm{Fe} / \mathrm{H}]<-1.0 \mathrm{dex})$ form a plateau at $[\alpha / \mathrm{Fe}] \sim 0.4$ dex. With

\footnotetext{
* Based on observations made with ESO telescopes at the La Silla Paranal Observatory under programmes ID 091.B-0171(A).

$\star \star$ The reduced spectra and the best fit synthetic models are available at the CDS via anonymous ftp to

cdsarc.u-strasbg.fr (130.79.128.5) or via

http://cdsarc.u-strasbg.fr/viz-bin/qcat?J/A+A/577/A18

$\star \star \star$ Member of the International Max Planck Research School for Astronomy and Cosmic Physics at the University of Heidelberg, IMPRS-HD, Germany.
}

the onset of SNe Ia, at metallicities above $[\mathrm{Fe} / \mathrm{H}] \sim-1.0 \mathrm{dex}$, the $[\alpha / \mathrm{Fe}]$ ratio starts to gradually drop, reaching solar values (McWilliam 1997). In dwarf galaxies that experienced slower star formation, lower values of $[\alpha / \mathrm{Fe}]$ are observed even at low metallicities owing to the lower enrichment rate of the interstellar medium from SNe II (see Shetrone et al. 2001, 2003; Venn et al. 2004; Tolstoy et al. 2009; Hendricks et al. 2014, and references therein). Although different $\alpha$ elements are produced on similar timescales, they show element-to-element scatter that is due to different production mechanisms, either through He burning in the cores of massive stars or during the SNe explosions themselves. In particular, sulphur (along with $\mathrm{Si}, \mathrm{Ar}$, and $\mathrm{Ca}$ ) is produced during both the $\mathrm{O}$-shell burning and explosive oxygenburning phases (Limongi \& Chieffi 2003).

Sulphur is an especially interesting $\alpha$-element because it is not depleted onto dust (Ueda et al. 2005) and is thus a genuine tracer of the "true" S abundance of the interstellar medium (ISM) and the stellar photospheres. In fact, sulphur is a preferred tracer of the $\alpha$ abundance in interstellar gas (Garnett 1989; Savage \& Sembach 1996) and in high-redshift star-forming environments such as damped Ly- $\alpha$ systems (Centurión et al. 2000; Nissen et al. 2004; Bowen et al. 2005). The existing sulphur measurements in halo field stars show that the $[\mathrm{S} / \mathrm{Fe}]$ ratio is increasing 
with decreasing $[\mathrm{Fe} / \mathrm{H}]$ at higher metallicities, reaching an average value of about 0.4 dex, but its behaviour at lower metallicities is still rather controversial. Different studies report either a bimodality of the $[\mathrm{S} / \mathrm{Fe}]$ ratio with a flat plateau and a branch of increasing abundance, reaching $[\mathrm{S} / \mathrm{Fe}] \sim 0.8 \mathrm{dex}$, or a plateau with a large scatter (see e.g. Nissen et al. 2004, 2007; Caffau et al. 2005a; Spite et al. 2011; Jönsson et al. 2011; Matrozis et al. 2013, and references therein).

Globular clusters (GC) represent the oldest stellar populations in the Milky Way and are examples of very dense environments. For a long time they were considered simple stellar populations, but today we recognize their complex SFH through precise abundance analysis of a variety of chemical elements in individual cluster member stars. All GCs studied to date present significant spreads and certain unique correlations in their light and $\alpha$ element abundances - generally taken as a clue for multiple enrichment phases (see the recent review by Gratton et al. 2012). Still, sulphur abundances have been derived in very few GCs because of the difficulties that such measurements entail. Sulphur lines in the optical spectral range are generally very weak in giant stars. At the same time, these brighter stars are often the only accessible tracers in remote and faint GCs. To date, the strong multiplet $1(920 \mathrm{~nm})$ has been used to obtain sulphur abundances in GCs (Caffau et al. 2005b, 2014; Sbordone et al. 2009; Koch \& Caffau 2011), but these lines are often blended with telluric absorption lines, and one of them is positioned in the blue wing of the Paschen $\zeta$ line and thus cannot always be reliably measured. This makes the Mult. 3 at $1045 \mathrm{~nm}$ particularly suited for measuring the sulphur abundance. Even if the lines are not as strong as the components of Mult. 1, a great advantage is that there are no telluric features in this wavelength range. Observing the $1045 \mathrm{~nm}$ sulphur lines leads to reliable results even at lower metallicities and will give independent and possibly more accurate sulphur abundances in GCs than using Mult. 1 (Caffau et al. 2007, 2010; Jönsson et al. 2011).

The metal-rich GC Ter $7([\mathrm{Fe} / \mathrm{H}]=-0.32 \mathrm{dex})$ is the first $\mathrm{GC}$ ever for which $\mathrm{S}$ abundances were derived. The three stars measured by Caffau et al. (2005b) are also the only extragalactic stars with a known $[\mathrm{S} / \mathrm{Fe}]$ ratio because Ter 7 is associated with the disrupted Sagittarius dwarf spheroidal galaxy. Caffau et al. (2005b) determined mean $\mathrm{S}$ abundance in Ter 7 to be slightly lower (by $\sim 0.1 \mathrm{dex}$ ) than in Galactic stars at similar metallicity. Sbordone et al. (2009) analysed nine stars in the GC 47 Tuc $([\mathrm{Fe} / \mathrm{H}]=-0.72 \mathrm{dex})$ and four stars in NGC 6752 $([\mathrm{Fe} / \mathrm{H}]=-1.54 \mathrm{dex})$, deriving $[\mathrm{S} / \mathrm{Fe}]=0.18 \pm 0.14$ and $[\mathrm{S} / \mathrm{Fe}]=$ $0.49 \pm 0.15$ dex for the two GCs, respectively, which is consistent with the results of field stars. Curiously, Sbordone et al. (2009) reported a star-to-star variation in the observed [S/Fe] ratio in 47 Tuc, which, although not significant with respect to the large measurement uncertainties, showed a strikingly clear correlation with the $\mathrm{Na}$ abundance. The authors considered different possibilities for the occurrence of this correlation, including that S may be involved in the self-enrichment processes of GCs through the ${ }^{31} \mathrm{P}(\mathrm{p}, \gamma){ }^{32} \mathrm{~S}$ proton capture reaction. Koch \& Caffau (2011) analysed a single star in the metal poor GC NGC 6397 $([\mathrm{Fe} / \mathrm{H}]=-2.0 \mathrm{dex})$, determining $[\mathrm{S} / \mathrm{Fe}]=0.52 \pm 0.2 \mathrm{dex}$, consistent with the halo field population. We also note the recent work by Caffau et al. (2014), which provides [S/Fe] measurements for several metal-rich open clusters and includes the GCM 4 that is analysed in the present work.

Here, we present the homogeneous analysis of 15 bright red giant branch (RGB) stars in three different GCs that together cover a wide range of metallicities $(-2.3<[\mathrm{Fe} / \mathrm{H}]<-1.2 \mathrm{dex})$. This strategy can provide answers to several basic questions. It is interesting to find out whether a dichotomy or a large spread of the $[\mathrm{S} / \mathrm{Fe}]$ ratio, as observed in the halo field stars, can also be found in GCs. The generally large uncertainties of the $[\mathrm{S} / \mathrm{Fe}]$ measurements in the halo field stars are mostly driven by uncertainties of the atmospheric parameters (Matrozis et al. 2013), and tracing the $[\mathrm{S} / \mathrm{Fe}]$ ratio with $\mathrm{GCs}$ will therefore lead to more accurate results because of the much better constrained distances, gravities, and effective temperatures of GC stars from colourmagnitude diagrams (CMD). Additionally, our data will help to check if there are star-to-star variations of the $\mathrm{S}$ abundance in the different GCs and whether they correlate with the other $\alpha$ and light element abundances, such as $\mathrm{O}, \mathrm{Na}, \mathrm{Si}, \mathrm{Mg}$, and $\mathrm{Al}$.

The three selected clusters are amongst those closest to the Sun that cover an optimally broad metallicity range and are all well-studied objects. The proximity and simple CMDs of M 4 and M30 make them desirable candidates for many observational and theoretical studies that test the current stellar evolutionary models. M 30 is also notable for its very low metallicity $[\mathrm{Fe} / \mathrm{H}]=-2.3$ dex. M 22, on the other hand, is amongst the most massive GCs. It has a very complex CMD with prominent multiple populations and a wide metallicity spread $(-1.9<[\mathrm{Fe} / \mathrm{H}]<$ -1.6 dex; Da Costa et al. 2009; Marino et al. 2009). It is often considered as a remainder of a disrupted dwarf galaxy and will thus add another important comparison object to our study.

In this paper we present the largest compilation of sulphur abundance measurements in Galactic clusters to date.

\section{Target selection and observations}

We chose our targets from existing high-resolution surveys of these GCs in the visible band; M 4 stars were chosen from Marino et al. (2008), M 22 stars from Marino et al. (2009, 2011), M 30 stars from Carretta et al. (2009a,b). Our intention was to select bright RGB stars that belong to different populations in the selected GCs. To this end, we targeted stars with different $\mathrm{Na}$ and $\mathrm{O}$ abundances in M 4 and M30. M22, on the other hand, is known to harbour stars with a wide metallicity spread, so we selected stars with different $[\mathrm{Fe} / \mathrm{H}]$. The adopted stellar parameters of the observed stars from the literature are presented in Table 1. Choosing stars for which high-resolution spectroscopy was already available is also important for constraining their atmospheric parameters, such as effective temperature, gravity, microturbulence velocity, and metallicity, which are necessary to determine the abundance. This is only possible if a large set of Fe I and Fe II lines is available, which is not the case with the narrow wavelength coverage of our spectra. Note, however, that the effective temperatures and gravities of the three stars in M 30 were determined photometrically by Carretta et al. (2009b) and most likely have larger uncertainties. We still opted to rely on them since these parameters were used by Carretta et al. (2009b) to derive abundances of other chemical elements in these stars, with which we aim to compare our $\mathrm{S}$ abundance estimates.

To compare the two approaches, we compared the stellar parameters for $\sim 80$ stars in the GC M4 in common between the studies of Marino et al. (2008) and Carretta et al. (2009b). The effective temperatures determined by Carretta et al. (2009b) are on average lower by $\sim 100 \mathrm{~K}$ than those in Marino et al. (2008) with $\sigma=45 \mathrm{~K}$ and the $\log g$ values used by Carretta et al. (2009b) are on average lower by 0.3 dex with $\sigma=0.15$ dex. Marino et al. (2008) find a lower spread in microturbulence and a wider spread in metallicities among the common stars, while Carretta et al. (2009b) find the opposite, that is, a wider spread in microturbulence and a lower spread in metallicity. The average 
Table 1. Atmospheric parameters and $[\mathrm{S} / \mathrm{Fe}]$ ratios of the targeted stars.

\begin{tabular}{|c|c|c|c|c|c|c|c|c|c|c|}
\hline ID & $\begin{array}{l}T_{\text {eff }} \\
{[\mathrm{K}]}\end{array}$ & $\begin{array}{l}\log g \\
\text { [dex] }\end{array}$ & $\begin{array}{c}v_{\text {mic }} \\
\mathrm{km} \mathrm{s}^{-1}\end{array}$ & $\begin{array}{c}{[\mathrm{Fe} / \mathrm{H}]} \\
{[\mathrm{dex}]}\end{array}$ & $\begin{array}{c}{[\mathrm{Na} / \mathrm{Fe}]} \\
{[\mathrm{dex}]}\end{array}$ & $\begin{array}{c}{[\mathrm{O} / \mathrm{Fe}]} \\
{[\mathrm{dex}]}\end{array}$ & $\begin{array}{c}{[\mathrm{Mg} / \mathrm{Fe}]} \\
{[\mathrm{dex}]}\end{array}$ & $\begin{array}{c}{[\mathrm{Ca} / \mathrm{Fe}]} \\
{[\mathrm{dex}]}\end{array}$ & $\begin{array}{c}v_{\mathrm{mac}}{ }^{d} \\
\mathrm{~km} \mathrm{~s}^{-1}\end{array}$ & $\begin{array}{c}{[\mathrm{S} / \mathrm{Fe}]_{\mathrm{LTE}}{ }^{e}} \\
{[\mathrm{dex}]}\end{array}$ \\
\hline \multicolumn{11}{|c|}{$\mathbf{M} 4^{a}$} \\
\hline 19925 & 4050 & 1.20 & 1.67 & -1.02 & $0.51 \pm 0.04$ & $0.28 \pm 0.04$ & $0.43 \pm 0.06$ & $0.19 \pm 0.03$ & $6.5 \pm 0.1$ & $0.57 \pm \mathbf{0 . 0 2}$ \\
\hline 21191 & 4270 & 1.60 & 1.60 & -1.06 & $0.51 \pm 0.04$ & $0.34 \pm 0.04$ & $0.55 \pm 0.06$ & $0.24 \pm 0.03$ & $4.8 \pm 0.1$ & $\mathbf{0 . 5 8} \pm \mathbf{0 . 0 2}$ \\
\hline 27448 & 4310 & 1.57 & 1.58 & -1.12 & $0.11 \pm 0.04$ & $0.51 \pm 0.04$ & $0.50 \pm 0.06$ & $0.29 \pm 0.03$ & $4.9 \pm 0.1$ & $0.57 \pm \mathbf{0 . 0 1}$ \\
\hline 28103 & 3860 & 0.50 & 1.62 & -1.08 & $0.17 \pm 0.04$ & $0.50 \pm 0.04$ & $0.41 \pm 0.06$ & $0.16 \pm 0.03$ & $6.3 \pm 0.1$ & $0.65 \pm 0.02$ \\
\hline 34006 & 4320 & 1.67 & 1.61 & -1.06 & $0.44 \pm 0.04$ & $0.25 \pm 0.04$ & $0.52 \pm 0.06$ & $0.26 \pm 0.03$ & $5.7 \pm 0.1$ & $0.50 \pm 0.02$ \\
\hline \multicolumn{11}{|c|}{ M $22^{b}$} \\
\hline 200005 & 4000 & 0.05 & 2.02 & -1.94 & $-0.02 \pm 0.03$ & $0.40 \pm 0.04$ & $0.46 \pm 0.04$ & $0.24 \pm 0.02$ & $11.9 \pm 0.2$ & $0.78 \pm 0.01$ \\
\hline 200025 & 4100 & 0.67 & 1.80 & -1.62 & $0.26 \pm 0.03$ & $0.49 \pm 0.04$ & $0.44 \pm 0.04$ & $0.42 \pm 0.02$ & $8.5 \pm 0.2$ & $0.57 \pm \mathbf{0 . 0 2}$ \\
\hline 200031 & 4300 & 0.77 & 1.55 & -1.85 & $0.31 \pm 0.03$ & $0.18 \pm 0.04$ & $0.34 \pm 0.04$ & $0.20 \pm 0.02$ & $9.4 \pm 0.2$ & $0.51 \pm 0.02$ \\
\hline 200051 & 4260 & 0.90 & 1.60 & -1.63 & $0.73 \pm 0.03$ & $-0.05 \pm 0.04$ & $0.44 \pm 0.04$ & $0.40 \pm 0.02$ & $6.8 \pm 0.2$ & $\mathbf{0 . 5 8} \pm \mathbf{0 . 0 2}$ \\
\hline 200061 & 4430 & 1.05 & 1.70 & -1.78 & $-0.17 \pm 0.03$ & $0.34 \pm 0.04$ & $0.54 \pm 0.04$ & $0.28 \pm 0.02$ & $12.1 \pm 0.6$ & $0.61 \pm 0.03$ \\
\hline \multicolumn{11}{|c|}{$\begin{array}{r}-0.01 \pm 0.03 \\
\mathbf{M ~ 3 0}\end{array}$} \\
\hline 5783 & 4463 & 1.17 & 2.32 & -2.33 & $0.57 \pm 0.02$ & $0.16 \pm 0.12$ & $\cdots$ & $\cdots$ & $8.7 \pm 0.7$ & $0.57 \pm \mathbf{0 . 0 3}$ \\
\hline 10849 & 4365 & 0.96 & 2.34 & -2.40 & $0.65 \pm 0.04$ & $-0.29 \pm 0.20$ & $\ldots$ & $\ldots$ & $11.9 \pm 0.9$ & $0.86 \pm 0.04$ \\
\hline 11294 & 4258 & 0.41 & 2.14 & -2.37 & $0.07 \pm 0.07$ & $0.28 \pm 0.08$ & $0.51 \pm 0.20$ & $\ldots$ & $12.2 \pm 0.4$ & $0.53 \pm 0.02$ \\
\hline
\end{tabular}

Notes. ${ }^{(a)}$ IDs, atmospheric parameters, and abundances from Marino et al. (2008). The adopted solar values are as follows: $A(\mathrm{Fe})=7.48$, $A(\mathrm{Na})=6.32, A(\mathrm{O})=8.83, A(\mathrm{Mg})=7.55, A(\mathrm{Ca})=6.39 .{ }^{(b)}$ IDs, atmospheric parameters, and abundances from Marino et al. (2009). The adopted solar values are as follows: $A(\mathrm{Fe})=7.48, A(\mathrm{Na})=6.31, A(\mathrm{O})=8.83, A(\mathrm{Mg})=7.54, A(\mathrm{Ca})=6.39 .{ }^{(c)} \mathrm{IDs}$, atmospheric parameters, and abundances from Carretta et al. (2009a,b). The adopted solar values are as in Gratton et al. $(2003): A(\mathrm{Fe})=7.54, A(\mathrm{Na})=6.21, A(\mathrm{O})=8.79$, $A(\mathrm{Mg})=7.43, A(\mathrm{Ca})=6.27 .{ }^{(d)}$ The macroturbulence velocity $\left(v_{\text {mac }}\right)$ is a free parameter in the fitting procedure. ${ }^{(e)}$ The adopted solar values are $A(\mathrm{Fe})=7.50, A(\mathrm{~S})=7.16($ Caffau et al. 2011).

Table 2. Observing log.

\begin{tabular}{|c|c|c|c|c|}
\hline ID & $\begin{array}{c}V \\
{[\mathrm{mag}]}\end{array}$ & Date & $\begin{array}{c}\text { Exp. time } \\
{[\mathrm{s}]}\end{array}$ & $\begin{array}{r}S / N \\
{\left[\mathrm{px}^{-1}\right]}\end{array}$ \\
\hline \multicolumn{5}{|c|}{ M 4} \\
\hline 19925 & 11.04 & Jun. 23/24 2013 & $8 \times 60$ & 220 \\
\hline 21191 & 11.70 & Sep. 13/14 2013 & $8 \times 120$ & 230 \\
\hline 27448 & 11.73 & Sep. 13/14 2013 & $8 \times 120$ & 230 \\
\hline 28103 & 10.71 & Apr. 5/6 2013 & $6 \times 60$ & 240 \\
\hline 34006 & 11.87 & Sep. 6/7 2013 & $8 \times 120$ & 160 \\
\hline 36215 & 11.80 & $\begin{array}{c}\text { Sep. 6/7 } 2013 \\
\text { M } 22\end{array}$ & $8 \times 120$ & 140 \\
\hline 200005 & 10.92 & Jun. 19/20 2013 & $8 \times 60$ & 230 \\
\hline 200025 & 11.52 & Jun. 19/20 2013 & $12 \times 60$ & 190 \\
\hline 200031 & 11.64 & Jun. 19/20 2013 & $8 \times 120$ & 180 \\
\hline 200051 & 12.04 & Jun. 20/21 2013 & $10 \times 120$ & 140 \\
\hline 200061 & 12.21 & Aug. 9/10 2013 & $14 \times 180$ & 100 \\
\hline 200068 & 12.30 & $\begin{array}{c}\text { Aug. 10/11 } 2013 \\
\text { M } 30\end{array}$ & $16 \times 120$ & 170 \\
\hline $5783^{a}$ & 12.71 & Jul. 27/28 2013 & $8 \times 300$ & 140 \\
\hline $5783^{a}$ & 12.71 & Aug. 22/23 2013 & $8 \times 300$ & 140 \\
\hline 10849 & 12.55 & Jul. 27/28 2013 & $12 \times 300$ & 100 \\
\hline 11294 & 12.09 & Aug. 22/23 2013 & $6 \times 300$ & 220 \\
\hline
\end{tabular}

Notes. ${ }^{(a)}$ This star was observed in two nights. The stated $\mathrm{S} / \mathrm{N}$ is for the combined spectrum of all $16 \times 300 \mathrm{~s}$ exposures.

differences are $0.13 \mathrm{~km} \mathrm{~s}^{-1}$ in microturbulence and $0.13 \mathrm{dex}$ in $[\mathrm{Fe} / \mathrm{H}]$ between the two studies.

Our spectra were taken in service mode under Programme ID 091.B-0171(A) with the VLT cryogenic high-resolution infrared echelle spectrograph (CRIRES Kaeufl et al. 2004) mounted at the Nasmyth focus A of the UT1 telescope (Antu). We used the $0.4^{\prime \prime}$ slit, which provides a resolving power $R \sim 50000$ and a dispersion scale on the four parallel $512 \mathrm{px}$ CCD chips of $0.05 \AA \mathrm{px}^{-1}$. The S I multiplet 3 was centred on chip 3 . We used relatively short exposure times (between 30 and $300 \mathrm{~s}$ ) with a nodding cycle between each exposure. The total integration time was chosen to achieve a signal-to-noise ratio $S / N \sim 200$ per px in the region of the sulphur lines. In the nodding technique, each star is moved along the slit and exposed at two different positions. This allows for proper sky subtraction and removal of systematic effects, such as amplifier glow. The final combined spectra are the mean of all short exposures. The science targets were also used as guide stars for the adaptive optics system. The observing log is presented in Table 2.

For the data reduction, we used the standard CRIRES pipeline (version 2.3.1) provided by ESO. The pipeline allows dark current subtraction, bad pixel correction, sky subtraction, flat-field correction, non-linearity correction, wavelength calibration from a Th-Ar lamp, and an extraction and combination of the final 1D spectrum from the science images. We noticed that the wavelength calibration was suboptimal as a result of the very few Th-Ar lamp lines with sufficient brightness available in the observed region, so we additionally refined the wavelength solution using the available stellar lines in the spectra. Finally, the spectra were continuum normalised by fitting the continuum regions with a high order polynomial.

\section{Results}

\subsection{Spectral synthesis}

We derived the $\mathrm{S}$ abundance for each of the observed stars through a full spectral synthesis using the local thermodynamic equilibrium (LTE) code Spectroscopy Made Easy (SME; Valenti \& Piskunov 1996). We derived our line list from the VALD database (Kupka et al. 1999, 2000). The transition parameters of the lines in multiplet 3 and the Fe I line at $1047.0 \mathrm{~nm}$ are presented in Table 3. There is no severe line blending in the 
Table 3. Line parameters from VALD.

\begin{tabular}{cccccccc}
\hline \hline Element & $\lambda$ & $\chi$ & $\log g f$ & \multicolumn{3}{c}{ Damping parameters } & Landé factor \\
& {$[\mathrm{nm}]$} & {$[\mathrm{eV}]$} & & radiative & stark & waals & \\
\hline $\mathrm{S} \mathrm{I}$ & $1045.5449^{a}$ & $6.8600^{a}$ & $+0.250^{a}$ & $8.950^{b}$ & $-5.370^{b}$ & $-7.333^{c}$ & $1.250^{b}$ \\
$\mathrm{Fe} \mathrm{I}$ & $1045.5404^{d}$ & $5.3930^{d}$ & $-0.905^{d}$ & $8.480^{d}$ & $-3.960^{d}$ & $-7.520^{d}$ & $1.130^{d}$ \\
$\mathrm{~S}$ I & $1045.6757^{a}$ & $6.8600^{a}$ & $-0.447^{a}$ & $8.950^{b}$ & $-5.370^{b}$ & $-7.333^{c}$ & $2.000^{b}$ \\
$\mathrm{~S}$ I & $1045.9406^{a}$ & $6.8600^{a}$ & $+0.030^{a}$ & $8.950^{b}$ & $-5.370^{b}$ & $-7.333^{c}$ & $1.750^{b}$ \\
$\mathrm{Fe} \mathrm{I}$ & $1046.9653^{d}$ & $3.8840^{d}$ & $-1.187^{d}$ & $6.830^{d}$ & $-6.150^{d}$ & $-7.820^{d}$ & $1.170^{d}$ \\
\hline
\end{tabular}

Notes. Line data references: ${ }^{(a)}$ Zerne et al. (1997); ${ }^{(b)}$ Kurucz (2004); ${ }^{(c)}$ Barklem et al. (2000); ${ }^{(d)}$ Kurucz (2007).

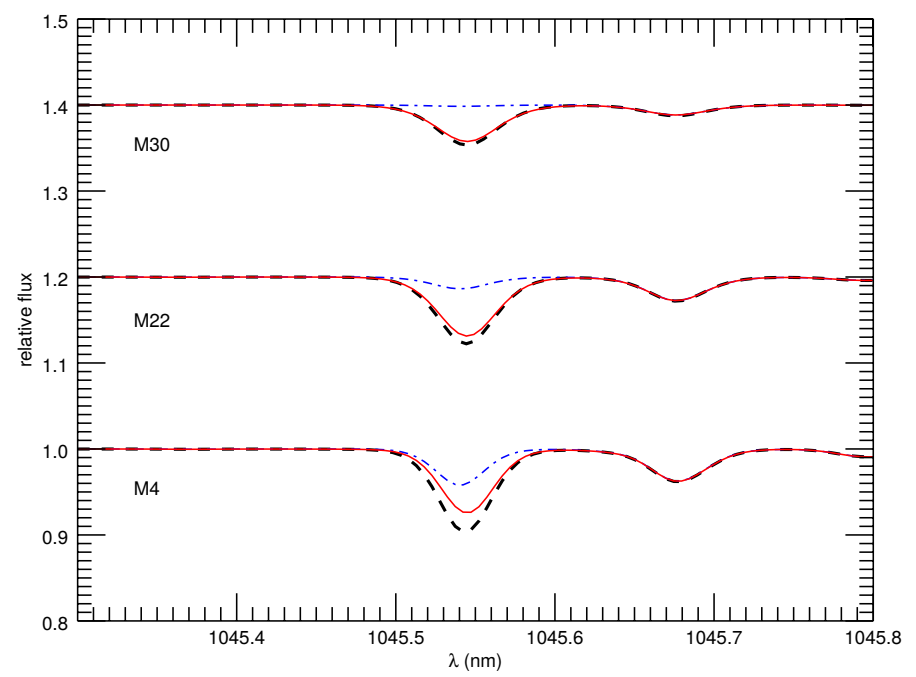

Fig. 1. Synthetic spectra illustrating the relative contribution of the Fe Iline (blue dashed-dotted line) at $1045.5 \mathrm{~nm}$ to the S I line at the same wavelength (red line) with respect to the combined spectral feature (thick dashed black line) at the metallicities of the three GCs assuming a solar abundance mix.

wavelength region of multiplet 3 . Only the S I line at $1045.3 \mathrm{~nm}$ is blended with a Fe I line, but the latter has a marginal contribution at $[\mathrm{Fe} / \mathrm{H}] \sim-1$ dex and practically disappears at lower metallicities. The relative contribution of this Fe I line lying on top of the S I feature is shown in Fig. 1 for the metallicities of the three GCs. The atomic data for this blending iron line is also given in Table 3. All other transitions on top of the $\mathrm{S}$ triplet are of a negligible strength, but we note that we used the complete line-list of the VALD database, including molecular bands in the respective wavelength interval in our synthesis. The region is free of telluric absorption lines. We interpolated the new grid of Kurucz ${ }^{1}$ plane-parallel, one-dimensional models without convective overshoot. These include the $\alpha$-enhanced opacity distribution functions (AODFNEW; Castelli \& Kurucz $2003)^{2}$. The models fully cover the parameter space of all stars in our sample with steps $\Delta T_{\text {eff }}=250 \mathrm{~K}, \Delta \log g=0.5$ dex, and $\Delta[\mathrm{Fe} / \mathrm{H}]=0.5$ dex. Kurucz models have also been used to derive the stellar parameters (Table 1). We discuss the impact of different atmospheric models on the $\mathrm{S}$ abundances in more detail in Sect. 3.2.

SME performs a $\chi^{2}$ minimisation algorithm iterating a defined set of free parameters. In our case, we used the $[\mathrm{S} / \mathrm{Fe}]$ ratio and the macroturbulence velocity of the stars as free parameters to simultaneously fit the three $\mathrm{S}$ lines from multiplet 3 and the

\footnotetext{
1 http://kurucz.harvard.edu/grids.html

2 http://wwwuser.oats.inaf.it/castelli/
}

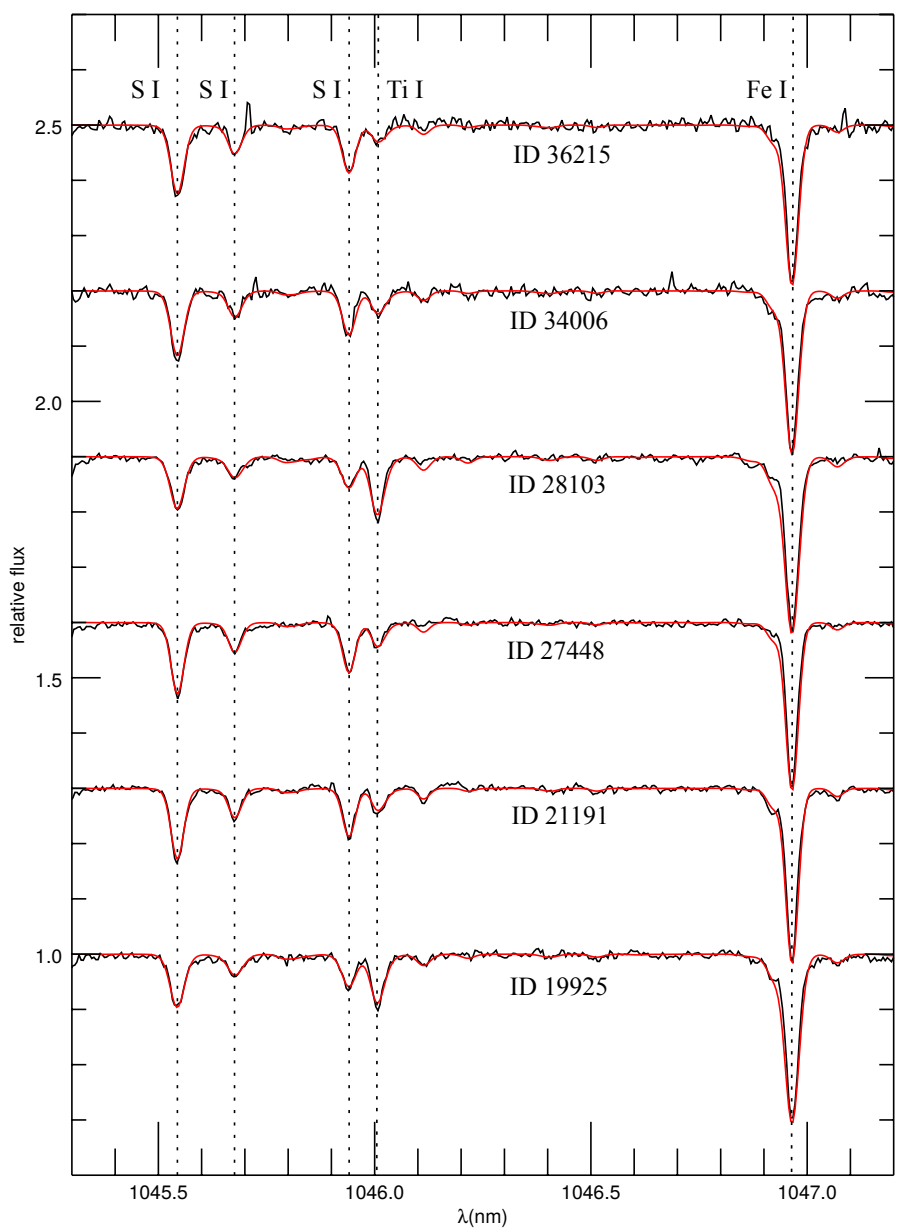

Fig. 2. Observed (black) and synthetic (red) spectra for the stars in M4. The most prominent spectral lines are marked with vertical dotted lines. The spectra are shifted vertically by an arbitrary value.

only prominent Fe line in the region (see Figs. 2-4). The stellar parameters, as presented in Table 1, were kept constant. The goodness of fit of the Fe I line shows the suitability of the input atmospheric parameters, since it is only affected by changes in the broadening parameter - the macroturbulence velocity in this case.

The estimated $[\mathrm{S} / \mathrm{Fe}]$ ratio in LTE and the best-fit values for the macroturbulence velocity for each star together with the statistical uncertainties are presented in the last two columns of Table 1 . We used the value of $A(\mathrm{~S})=7.16 \mathrm{dex}$ and $A(\mathrm{Fe})=$ $7.50 \mathrm{dex}$ as in Caffau et al. (2011) for the solar abundance of sulphur and iron, respectively.

We find very homogeneous $[\mathrm{S} / \mathrm{Fe}]$ ratios for all six stars in M 4 with a mean $[\mathrm{S} / \mathrm{Fe}]_{\mathrm{LTE}}=0.58 \pm 0.01 \mathrm{dex}$ with an intrinsic 
Table 4. Systematic uncertainties of $[\mathrm{S} / \mathrm{Fe}]$.

\begin{tabular}{ccccccccccc}
\hline \hline GC & \multirow{2}{*}{ Star ID } & \multicolumn{2}{c}{$\Delta T_{\text {eff }}$} & \multicolumn{2}{c}{$\Delta \log g$} & \multicolumn{2}{c}{$\Delta v_{\text {mic }}$} & \multicolumn{2}{c}{$\Delta[\mathrm{Fe} / \mathrm{H}]$} & \multicolumn{2}{c}{ Total } \\
& & $+100 \mathrm{~K}$ & $-100 \mathrm{~K}$ & $+0.1 \mathrm{dex}$ & $-0.1 \mathrm{dex}$ & $+0.1 \mathrm{~km} \mathrm{~s}^{-1}$ & $-0.1 \mathrm{~km} \mathrm{~s}^{-1}$ & $+0.05 \mathrm{dex}$ & $-0.05 \mathrm{dex}$ & \\
\hline M4 & 27448 & -0.18 & +0.20 & +0.04 & -0.05 & -0.01 & 0.00 & -0.03 & +0.03 & \pm 0.20 \\
M22 & 200051 & -0.18 & +0.18 & +0.05 & -0.06 & 0.00 & 0.01 & -0.05 & +0.03 & \pm 0.19 \\
M30 & 11294 & -0.14 & +0.16 & +0.05 & -0.04 & 0.00 & 0.01 & -0.02 & +0.03 & \pm 0.16 \\
\hline
\end{tabular}

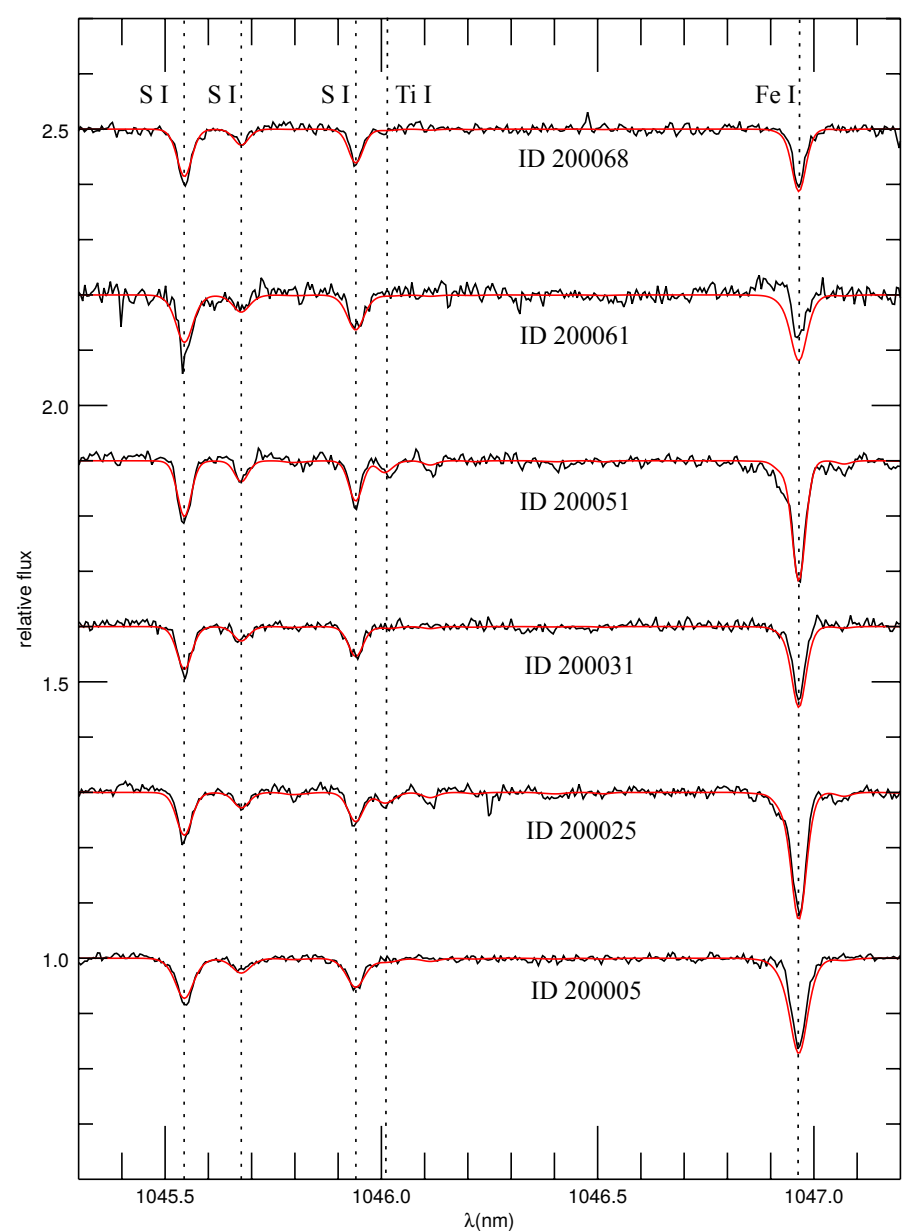

Fig. 3. Same as Fig. 2, but for the stars in M 22.

spread $\sigma_{0}=0.04$ dex. The intrinsic spread is calculated as $\sigma_{0}^{2}=\sigma_{[\mathrm{S} / \mathrm{Fe}]}^{2}-\left\langle\epsilon_{\text {rand }}\right\rangle^{2}$, where $\sigma_{[\mathrm{S} / \mathrm{Fe}]}$ is the standard deviation of the $[\mathrm{S} / \mathrm{Fe}]$ ratio from the mean of all stars and $\left\langle\epsilon_{\text {rand }}\right\rangle$ is the average random error of the individual measurements. The random errors $\left(\epsilon_{\text {rand }}\right)$ are the formal uncertainties based on the goodness of the $\chi^{2}$ fit. Five of the observed stars in M22, regardless of their metallicity, have consistent abundances with a mean $[\mathrm{S} / \mathrm{Fe}]_{\mathrm{LTE}}=0.57 \pm 0.01 \mathrm{dex}$ with an intrinsic spread $\sigma_{0}=0.03$ dex. There is only one star (ID 200005) that shows somewhat higher $[\mathrm{S} / \mathrm{Fe}]_{\mathrm{LTE}}=0.78 \pm 0.01 \mathrm{dex}$. The situation is similar for M30. Two stars show consistent $[\mathrm{S} / \mathrm{Fe}]$ ratios with a mean $[\mathrm{S} / \mathrm{Fe}]_{\mathrm{LTE}}=0.55 \pm 0.02$ dex and the third one (ID 10849) has an abnormally high $[\mathrm{S} / \mathrm{Fe}]_{\text {LTE }}=0.86 \pm 0.04$ dex. Note, however, that the derived $[\mathrm{S} / \mathrm{Fe}]$ ratios are strongly dependent on the effective temperatures and moderately on the gravities and metallicities of the stars (see Sect. 3.2). Thus, reasonable changes in the effective temperatures of the stars with abnormal $\mathrm{S}$ abundances can bring them in line with the other stars in the sample, for instance an increase of $T_{\text {eff }}$ by $100 \mathrm{~K}$ for star 200005

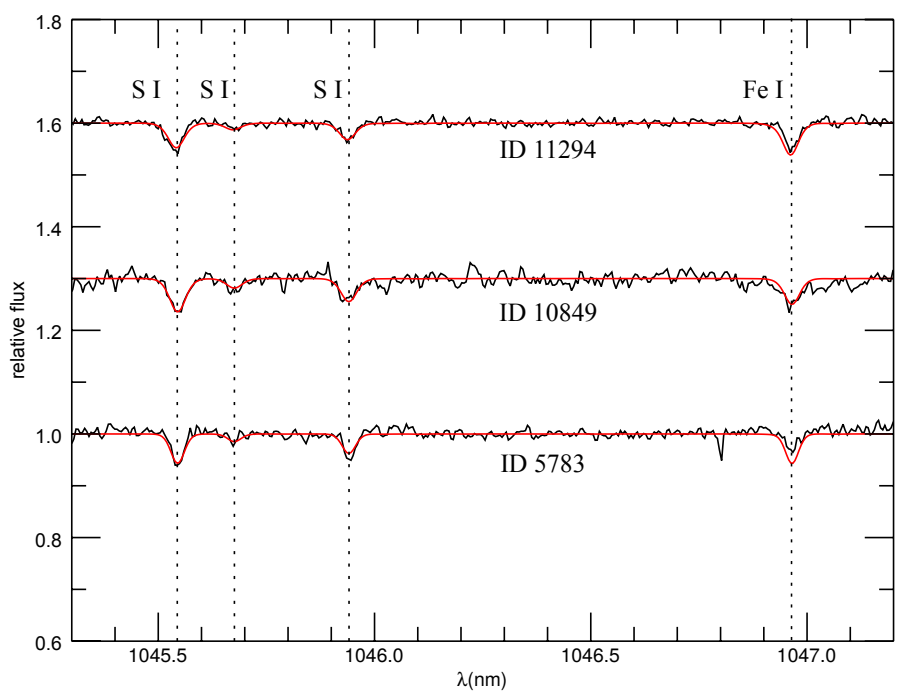

Fig. 4. Same as Fig. 2, but for the stars in M 30.

in M 22 and by $200 \mathrm{~K}$ for star 10849 in M30. Star 200005 also has a somewhat lower $[\mathrm{Fe} / \mathrm{H}]$ estimate $\sim 0.1$ dex lower than the mean of the metal-poor population of M 22 (Marino et al. 2009). As indicated in Table 4, increasing the metallicity of the star by 0.1 dex will bring the $[\mathrm{S} / \mathrm{Fe}]$ ratio down by $0.1 \mathrm{dex}$, which agrees reasonably well with the rest of the stars in M 22.

The reference Fe I line in our spectra for star 200005 is likewise only poorly fit by the synthetic spectrum, with a $\chi^{2}$ estimation about 2.5 times higher than the average for the other stars. It cannot be improved by only changing the macroturbulence velocity. This means that the model spectrum does not reproduce the equivalent width of the line correctly, which is another indication that the stellar parameters for this star might be incorrect. We are able to obtain very good fits to the iron line by modifying the effective temperature, gravity, and metallicity of the star but because of the degeneracy between these parameters, it is difficult to firmly conclude on its sulphur abundance. The situation with star 10849 in M 30 (it also has the lowest S/N spectrum in our sample) is more critical. As our comparison of the stellar parameter determination using the methodology of Carretta et al. (2009b) and Marino et al. (2008) for the common stars in M 4 showed, there are systematic differences, but the standard deviations in $T_{\text {eff }}$ and $\log g$ appear to be small. We do not have solid reasons to believe that the relative estimate of the effective temperature of a star in a homogeneous study could be incorrect by as much as $200 \mathrm{~K}$, and it does not present odd abundances of any other element (Carretta et al. 2009b).

\subsection{Systematic uncertainties}

To estimate the systematic errors of the $[\mathrm{S} / \mathrm{Fe}]$ ratio caused by uncertainties in the atmospheric parameters of the stars, we chose one star from each GC and varied its $T_{\text {eff }}$ by $\pm 100 \mathrm{~K}$, 


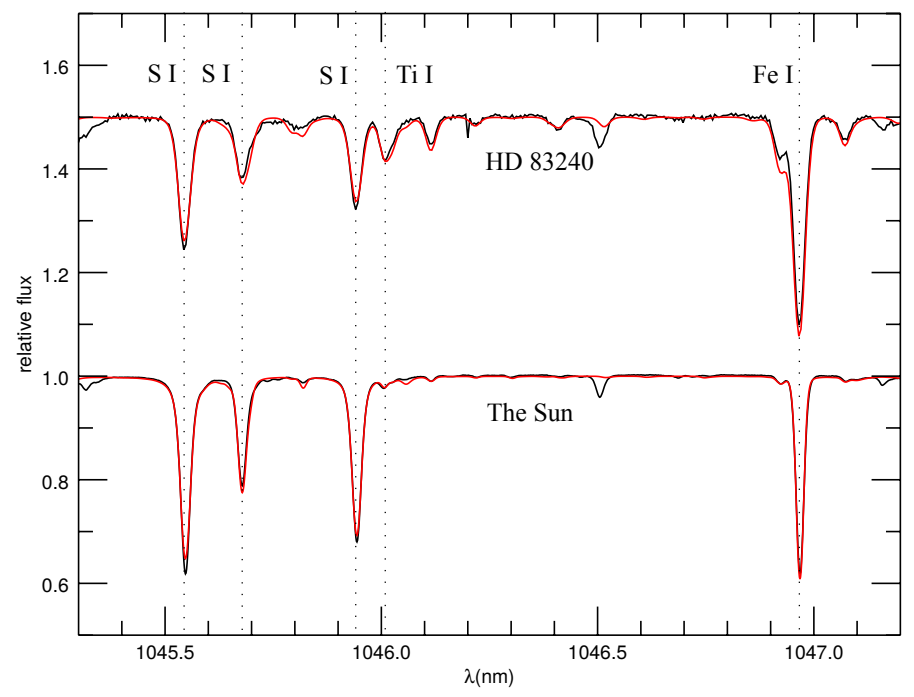

Fig. 5. The S multiplet 3 region in the Sun and the benchmark RGB star HD 83240 fitted using the same technique as for the GC stars. In the Sun, we estimate $A(\mathrm{~S})_{\text {LTE }}=7.26$ dex opposed to the adopted solar abundance of $A(\mathrm{~S})=7.16$ dex. For HD 83240 we find $A(\mathrm{~S})_{\text {LTE }}=7.13$ dex. Symbols are as in Fig. 2.

$\log g$ by \pm 0.1 dex, $v_{\text {mic }}$ by $\pm 0.1 \mathrm{~km} \mathrm{~s}^{-1}$, and $[\mathrm{Fe} / \mathrm{H}]$ by \pm 0.05 dex. These values are very similar to the uncertainties of the atmospheric parameters given in the literature. The results are presented in Table 4. In the last column, we list the total systematic error, which is calculated by combining in quadrature the uncertainties caused by different parameters. We note, however, that this is an upper limit of the systematic uncertainties due to covariances of the different parameters (see McWilliam et al. 1995).

The choice of the atmosphere structure also has only a marginal impact on the derived abundances. For instance, a solar-scaled Kurucz atmosphere model (ODFNEW) would lead to $\mathrm{S}$ abundances on average lower by 0.12 dex for the stars in M4. The differences are less pronounced in the more metalpoor GCs where the solar-scaled models would render $\mathrm{S}$ abundances lower by 0.07 dex and 0.04 dex for M 22 and M 30, respectively. We also tested the Marcs 2012 spherically symmetric models, which are $\alpha$-enhanced for metallicities below -1 dex (Gustafsson et al. 2008). These models lead to $\mathrm{S}$ abundances on average higher by 0.07 dex than the ones stated in Table 1 for all three GCs. All model grids are equally densely populated in the parameter space of interest.

\subsection{NLTE and 3D effects}

The $\mathrm{S}$ lines at $1045 \mathrm{~nm}$ are affected by relatively strong NLTE effects. By extrapolating the NLTE results from the grid of Takeda et al. (2005) to the stellar parameters of our stars, we found $\Delta_{\mathrm{NLTE}}=-0.20 \mathrm{dex}, \Delta_{\mathrm{NLTE}}=-0.15 \mathrm{dex}$, and $\Delta_{\mathrm{NLTE}}=-0.10 \mathrm{dex}$ for the GCs M4, M 22, and M30, respectively. The corrections become smaller for lower metallicities. This produces a weak trend of slightly increasing $[\mathrm{S} / \mathrm{Fe}]_{\text {NLTE }}$ ratios with decreasing metallicity with respect to the very homogeneous mean $[\mathrm{S} / \mathrm{Fe}]_{\mathrm{LTE}}$ values for the three GCs stated in Sect. 3.1. We find $[\mathrm{S} / \mathrm{Fe}]_{\text {NLTE }}=0.38 \mathrm{dex}, 0.42 \mathrm{dex}$, and 0.45 dex for the GC M 4, M 22, and M30, respectively - which agrees better with the nominal $[\alpha / \mathrm{Fe}]=0.4$ dex value for the old halo stellar population. Considering the large systematic uncertainties, the small increase of the $[\mathrm{S} / \mathrm{Fe}]_{\mathrm{NLTE}}$ ratios with decreasing metallicity is probably insignificant.

According to calculations by Jönsson et al. (2011), based on the 3D model atmospheres of Collet et al. (2007, 2009), the $3 \mathrm{D}$ corrections for $\mathrm{S}$ abundances derived from multiplet 3 are constant and positive at all metallicities for stars with similar parameters to ours and are in the order of $\Delta_{3 \mathrm{D}}=+0.20$ dex. Caffau et al. $(2007,2010)$ explored the 3D effects of multiplet 3 in dwarf stars and also found positive corrections based on the $\mathrm{CO}^{5} \mathrm{BOLD}$ 3D model atmospheres. Although it is inconsistent to apply their results to giant stars, there is an agreement that the 3D corrections for the lines at $1045 \mathrm{~nm}$ are always positive. In general, the NLTE and 3D corrections for our stars roughly compensate each other and are in the order of the systematic uncertainties. The 3D-LTE corrections need to be applied to 1D-NLTEcorrected results with caution, however, since the abundances derived from full 3D-NLTE analysis might differ substantially (see Sect. 3.4). For this reason, and to be consistent with literature $[\mathrm{S} / \mathrm{Fe}]$ measurements of field and cluster stars, we considered only the $[\mathrm{S} / \mathrm{Fe}]_{\mathrm{NLTE}}$ results stated above for the remaining discussion.

\subsection{Sulphur abundance of the Sun and HD 83240}

Finally, the $[\mathrm{S} / \mathrm{Fe}]$ ratio is given relative to the solar abundance of sulphur and therefore it is vital for the proper analysis. We decided to apply the same spectral synthesis technique to the same region of the Kurucz solar atlas (Kurucz et al. 1984) and derived the LTE solar abundance of sulphur estimated from multiplet 3 (Fig. 5). We found $A(\mathrm{~S})=7.26 \pm 0.01 \mathrm{dex}$, which is higher by 0.10 dex than the nominal value of $A(\mathrm{~S})=7.16 \mathrm{dex}$ (Caffau et al. 2011) adopted in this work. Our result is consistent with the solar value estimated by Caffau et al. (2007) using the same lines of multiplet $3(A(S)=7.30 \mathrm{dex})$. This finding systematically decreases our derived abundances in LTE in the three GCs by 0.1 dex. Although the 1D-NLTE and 3D corrections for the solar abundance derived from multiplet 3 are small and cancel each other out $-\Delta_{\text {NLTE }}=-0.07$ dex (Takeda et al. 2005 ) and $\Delta_{3 \mathrm{D}}=+0.07 \mathrm{dex}$ (Caffau et al. 2007), it is likely that an NLTE analysis performed in a full 3D synthesis will bring the abundance down to the nominal value. The nominal solar value is estimated from the $\mathrm{S}$ triplet at $675.7 \mathrm{~nm}$ and the [S I] line at $1082 \mathrm{~nm}$, which are virtually unaffected by NLTE effects (Caffau et al. 2007; Caffau \& Ludwig 2007).

To test the reliability of our results for RGB GC stars we also carried out an identical analysis for a well-studied giant benchmark star - HD 83240. We used a CRIRES spectrum from the library of Lebzelter et al. (2012) ${ }^{3}$. HD 83240 is classified as a K1 III star and has a $T_{\text {eff }}=4680 \mathrm{~K}, \log g=2.45 \mathrm{dex}$, $v_{\text {mic }}=1.3 \mathrm{~km} \mathrm{~s}^{-1}$ and $[\mathrm{Fe} / \mathrm{H}]=-0.02 \mathrm{dex}$. Although no sulphur abundance was explicitly derived for this star, Mishenina et al. $(2006,2007)$ measured the abundances of various light, Fe-peak, and n-capture elements and found that the star has a solar abundance pattern with a slight under-abundance of carbon and over-abundance of nitrogen, consistent with a post firstdredge-up evolutionary stage. Thus, we assume that HD 83240 also has a solar abundance of sulphur. We measured $[\mathrm{S} / \mathrm{Fe}]_{\mathrm{LTE}}=$ -0.01 dex (Fig. 5). The NLTE correction for this star is expected to be in the order of -0.15 dex, and the $3 \mathrm{D}$ correction is positive and in the same order of magnitude.

http://www.univie.ac.at/crirespop/index.html 

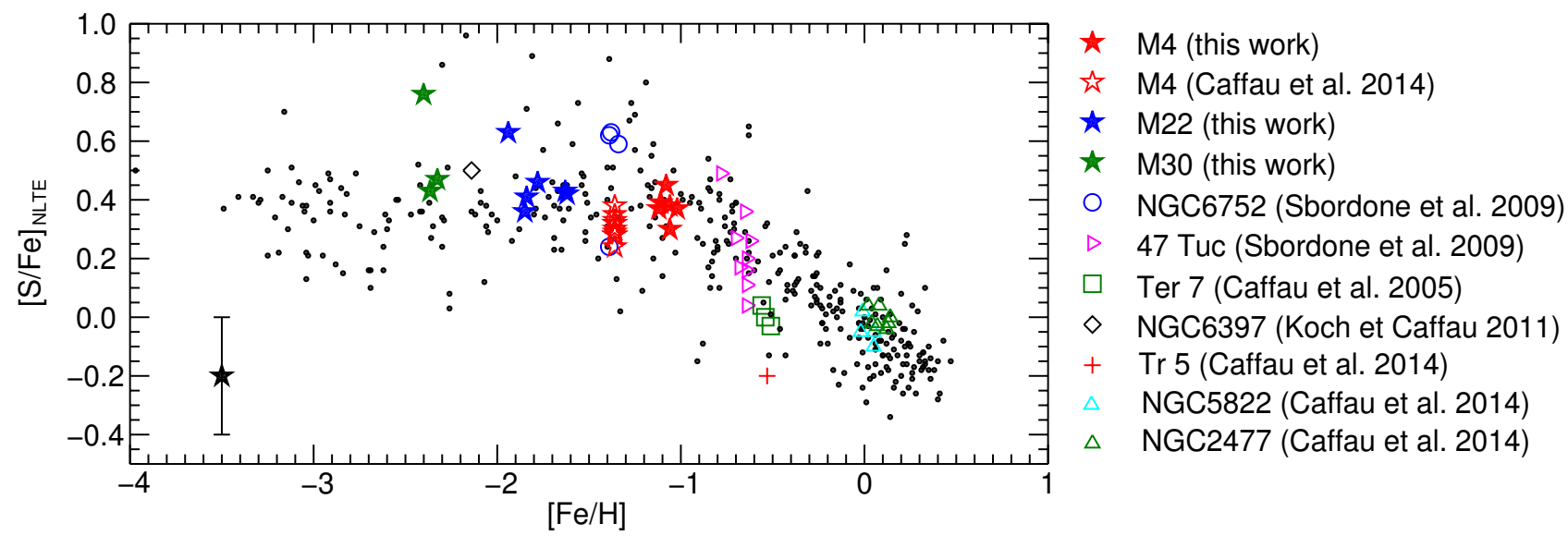

Fig. 6. NLTE-corrected [S/Fe] abundance ratios as a function of metallicity in Galactic disk and halo stars (black dots: Caffau et al. 2005a; Jönsson et al. 2011; Takeda \& Takada-Hidai 2011, 2012; Spite et al. 2011) and cluster stars (coloured symbols). All abundances are referenced to the adopted solar value of $A(S)=7.16 \mathrm{dex}$. The random errors of our GC stars are comparable to the symbol sizes. The maximum systematic error due to uncertainties of the stellar parameters is shown in the figure.

The Fe I line that we used to adjust the broadening in our spectra is well reproduced by our synthesis in both the Sun and HD 83240. Thus we confirm the suitability of the atomic data for this line.

\section{Implications on the Galactic chemical evolution of sulphur}

We should be careful when directly comparing the stellar [S/Fe] abundance ratios from different studies because they are based on different methods, different $\mathrm{S}$ lines, and different assumptions in their final results. It has been shown that even after proper NLTE treatment, the various $S$ features can result in offsets in the derived abundances (see Jönsson et al. 2011). Nevertheless, in Fig. 6 we have compiled a large collection of known [S/Fe] ratios (references are provided in the figure legend and caption) for Galactic and cluster stars to roughly track the evolution of sulphur. All measurements have been shifted to the solar value of sulphur $(A(S)=7.16$ dex $)$ adopted in this work, and NLTE corrections are applied where applicable. Most of the NLTE corrections come from the calculations of Takeda et al. (2005), except for the extremely metal-poor stars of Spite et al. (2011), who computed their own NLTE corrections. We note that although the NLTE results by both teams are computed for different types of stars, there are some differences in the overlapping regime. We did not apply 3D corrections in the results plotted in Fig. 6 to be consistent with most of the literature discussions.

There are $\mathrm{S}$ abundances derived within the entire span of metallicities covered by stars in Galactic clusters, although the sample is still limited and further studies are desirable. In all analysed clusters, sulphur seems to behave as a typical $\alpha$ element, meaning that the stars in metal poor Galactic GCs $([\mathrm{Fe} / \mathrm{H}]<-1.0$ dex $)$ have enhanced $[\mathrm{S} / \mathrm{Fe}]$ abundance ratios and open clusters of solar metallicity have solar $[\mathrm{S} / \mathrm{Fe}]$ ratios, consistent with the results for field stars and chemical evolutionary models. The only exceptions are $\operatorname{Tr} 5$ discussed in Caffau et al. (2014) and Ter 7 (Caffau et al. 2005b) with anomalously low $[\mathrm{S} / \mathrm{Fe}]$ ratios. The latter is associated with the Sagittarius dwarf spheroidal galaxy and its low $[\mathrm{S} / \mathrm{Fe}]$ ratio is consistent with the lower star formation efficiency of its parent galaxy. While the spread of sulphur on the metal poor plateau as traced by cluster stars is smaller on average than the spread traced by Galactic field stars, we found one star in M 22 and one in M 30 that are compatible with extreme $\mathrm{S}$ abundances.

Messier 4 is the only GC analysed by two teams independently (Caffau et al. 2014, and this work) and a more careful comparison between the results is possible. Caffau et al. (2014) derived $\mathrm{S}$ abundances for ten stars using line profile fitting of multiplet 1 at $920 \mathrm{~nm}$ taking into account the telluric absorption lines as well. Although both studies seem to agree on consistent $[\mathrm{S} / \mathrm{Fe}]$ abundance ratios for this $\mathrm{GC}$ (see Fig. 6), Caffau et al. (2014) assumed a lower metallicity for their stars and adopted $[\mathrm{Fe} / \mathrm{H}]=-1.36$ dex for $\mathrm{M} 4$ (Monaco et al. 2012), while the mean $[\mathrm{Fe} / \mathrm{H}]$ for our stars is $=-1.08$ dex (Marino et al. 2008). This discrepancy results in a much lower value of $A(S)=6.11 \pm 0.04$ dex estimated by Caffau et al. (2014), compared to our estimate of $A(\mathrm{~S})=6.46 \pm 0.02 \mathrm{dex}$ in NLTE. The NLTE corrections for both multiplets are similar and in the order of 0.2 dex (Takeda et al. 2005). Both studies agree, however, that there is no significant spread in $[\mathrm{S} / \mathrm{Fe}]$ amongst the RGB stars in M 4.

Finally, we explore the behaviour of sulphur with respect to other light and $\alpha$ elements. One of the goals of this study is to probe whether star-to-star variations within an individual GC are possible, as is the case for elements such as $\mathrm{C}, \mathrm{N}, \mathrm{O}, \mathrm{Na}, \mathrm{Al}$, and to some extent $\mathrm{Mg}$ and $\mathrm{Si}$, which are all correlated (see the review by Gratton et al. 2012, and references therein). In Fig. 7 (left panel), we plot existing measurements of $[\mathrm{S} / \mathrm{Fe}]$ vs. $[\mathrm{Na} / \mathrm{Fe}]$ for various GCs. In M 4, M 22, and M30, we have deliberately targeted stars with different $\mathrm{Na}$ abundances, and Fig. 7 clearly shows that there is no S-Na correlation between stars from these GCs. While the high S star in M 30 is also Na-rich, the high S star in M 22 has a low Na abundance. So far, with the only tentative exception of 47 Tuc that was previously discussed in Sbordone et al. (2009), it seems that even if present, star-to-star variations of the sulphuric abundance in GCs are not caused by the same self-enrichment processes that are responsible for the formation of p-capture element abundance variations. We note, however, that we still lack an extensive study that includes a large number of stars in a single GC.

In the middle panel of Fig. 7 we show the behaviour of the $[\mathrm{S} / \mathrm{Ca}]$ ratio in GCs - two $\alpha$ elements carved through the same production channels. The mean ratio is $\sim 0.1$ dex, and there is no trend with metallicity, which suggests that the two elements do evolve in lock-step, as expected. There is more scatter in the 

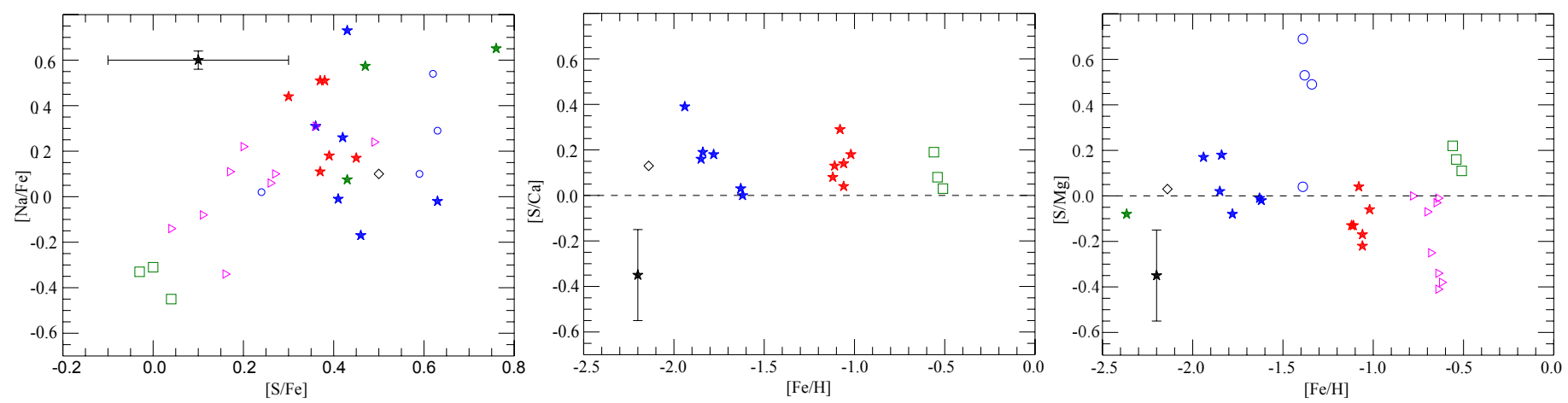

Fig. 7. Comparison of the $\mathrm{S}$ abundances with the abundances of other light and $\alpha$ elements in $\mathrm{GCs}$ : $[\mathrm{S} / \mathrm{Fe}] \mathrm{vs}$. $[\mathrm{Na} / \mathrm{Fe}]($ left panel); $[\mathrm{Fe} / \mathrm{H}]$ vs. $[\mathrm{S} / \mathrm{Ca}]$ (middle panel); $[\mathrm{Fe} / \mathrm{H}]$ vs. $[\mathrm{S} / \mathrm{Mg}]$ (right panel). Different symbols indicate stars from different clusters as indicated in the legend of Fig. 6. The maximum systematic errors are also indicated.

[S/Mg] ratio, although the mean value is 0 dex (Fig. 7, right panel). Both elements have slightly different production chains, so the larger scatter is not surprising. In the pre-supernova evolution, $\mathrm{Mg}$ is produced in the $\mathrm{C}$-convective shell burning, but $\mathrm{S}$ - in the O-convective shell burning. During the explosive nucleosynthesis, $\mathrm{Mg}$ is produced in $\mathrm{C}$ and $\mathrm{Ne}$ burning, but $\mathrm{S}$ - in $\mathrm{O}$ burning (Limongi \& Chieffi 2003). Magnesium could also participate in p-capture reactions through the $\mathrm{MgAl}$ hydrogen-burning cycle at high temperatures, and hence star-to-star variations of this element are prominent in some GCs. While in a majority of the observed GC stars that cover a wide range of metallicities, the behaviour of sulphur with respect to other $\alpha$ elements favours a typical $\alpha$-capture origin of this element, the detection of two very S-rich stars (if confirmed real) may raise the question of additional production channels.

\section{Conclusions}

Suitable lines for measuring sulphur in the visible band at low metallicities are rare, therefore the $\mathrm{S}$ abundance measurements in GC stars are quite scarce to date. The present work helps to substantially increase the sample of cluster stars with determined $\mathrm{S}$ abundances. We measured the $[\mathrm{S} / \mathrm{Fe}]$ abundance ratio from multiplet 3 at $1045 \mathrm{~nm}$ in 15 RGB stars from three GCs (M4, M 22, and M30) observed with the high-resolution, infrared spectrograph CRIRES, mounted at the VLT. Multiplet 3 is suited for $\mathrm{S}$ abundance derivation since the three lines are relatively strong even at low metallicity and are free of significant stellar blends and telluric contamination. The selected GCs cover a wide range of metallicities $(-2.3<[\mathrm{Fe} / \mathrm{H}]<-1.0 \mathrm{dex})$, and the individual RGB targets were especially selected to have different $\mathrm{Na}$ and $\mathrm{O}$ abundances, or even different $\mathrm{Fe}$ abundances in the case of the massive GC M 22. This ensured that we have stars belonging to different cluster populations, and it allowed us to search for possible star-to-star variations of the $\mathrm{S}$ abundance in GCs.

We find that the $\mathrm{S}$ abundances derived from multiplet 3 are very sensitive to changes in the effective temperature of the observed stars, and the uncertainty of this parameter dominates the uncertainty of the measured abundances. The lines of multiplet 3 are also prone to large departures from LTE and are sensitive to horizontal fluctuations of the stellar atmosphere, inferring large 3D model corrections. The NLTE and 3D corrections are similar in magnitude and roughly compensate each other, but because we lack a full NLTE analysis performed within the framework of a 3D atmospheric model, additional systematic uncertainty is introduced. Nevertheless, we perceive very small star-to-star scatter in most of the observed stars in the three GCs, which is consistent with a narrow plateau $\left([\mathrm{S} / \mathrm{Fe}]_{\mathrm{LTE}}=0.57 \pm 0.01 \mathrm{dex}\right.$, $\left.\sigma_{0}=0.03 \mathrm{dex} ;[\mathrm{S} / \mathrm{Fe}]_{\mathrm{NLTE}}=0.40 \mathrm{dex}, \sigma_{0}=0.05 \mathrm{dex}\right)$, as expected for the behaviour of a typical $\alpha$ element at low metallicity in the Milky Way. Sulphur appears to vary in lock-step with $\mathrm{Ca}$, a well-studied $\alpha$ element most likely produced through the same nuclear channels. The mean $[\mathrm{S} / \mathrm{Mg}]$ ratio is solar, but with a larger scatter due to the more complex production path of $\mathrm{Mg}$, which could also participate in the self-enrichment processes that take place in GCs.

One star in the GC M22 and one star in the GC M30 are found to be exceptionally $\mathrm{S}$ rich, which is consistent with the high $\mathrm{S}$ measurements in some field stars (e.g. Caffau et al. 2005a). We considered the possibility that these are erroneous measurements due to some deviation from the true atmospheric parameters of the two stars, but we cannot unambiguously exclude the possibility of observing genuine S-rich stars in GCs. The S-rich stars do not show clear correlations with p-capture elements like $\mathrm{Na}$ and $\mathrm{O}$, therefore we conclude that $\mathrm{S}$ is not produced during the hot hydrogen-burning reactions that are responsible for the formation of multiple population in GCs. Discovering stars with confirmed extreme $[\mathrm{S} / \mathrm{Fe}]$ ratios in different stellar environments would require the invention of new production mechanisms for sulphur.

Clearly, more observations are needed, in particular large homogeneous samples, to conclusively constrain the $\mathrm{S}$ abundances in GCs.

Acknowledgements. We thank N. Piskunov and G. Ruchti for help with the SME code and A. O. Thygesen and H.-G. Ludwig for insightful discussions. N.K. and A.K. acknowledge the Deutsche Forschungsgemeinschaft for funding from Emmy-Noether grant Ko 4161/1. E.C. is grateful to the FONDATION MERAC for funding her fellowship.

\section{References}

Barklem, P. S., Piskunov, N., \& O’Mara, B. J. 2000, A\&AS, 142, 467 Bowen, D. V., Jenkins, E. B., Pettini, M., \& Tripp, T. M. 2005, ApJ, 635, 880 Caffau, E., \& Ludwig, H.-G. 2007, A\&A, 467, L11

Caffau, E., Bonifacio, P., Faraggiana, R., et al. 2005a, A\&A, 441, 533 Caffau, E., Bonifacio, P., Faraggiana, R., \& Sbordone, L. 2005b, A\&A, 436, L9 Caffau, E., Faraggiana, R., Bonifacio, P., Ludwig, H.-G., \& Steffen, M. 2007, A\&A, 470, 699

Caffau, E., Sbordone, L., Ludwig, H.-G., Bonifacio, P., \& Spite, M. 2010, Astron. Nachr., 331, 725

Caffau, E., Ludwig, H.-G., Steffen, M., Freytag, B., \& Bonifacio, P. 2011, Sol. Phys., 268, 255

Caffau, E., Monaco, L., Spite, M., et al. 2014, A\&A, 568, A29

Carretta, E., Bragaglia, A., Gratton, R., \& Lucatello, S. 2009a, A\&A, 505, 139

Carretta, E., Bragaglia, A., Gratton, R. G., et al. 2009b, A\&A, 505, 117

Castelli, F., \& Kurucz, R. L. 2003, IAUS, 210, 20 
Centurión, M., Bonifacio, P., Molaro, P., \& Vladilo, G. 2000, ApJ, 536, 540

Collet, R., Asplund, M., \& Trampedach, R. 2007, A\&A, 469, 687

Collet, R., Nordlund, A., Asplund, M., Hayek, W., \& Trampedach, R. 2009, Mem. Soc. Astron. It., 80, 719

Da Costa, G. S., Held, E. V., Saviane, I., \& Gullieuszik, M. 2009, ApJ, 705, 1481 Garnett, D. R. 1989, ApJ, 345, 282

Gratton, R. G., Carretta, E., Claudi, R., Lucatello, S., \& Barbieri, M. 2003, A\&A, 404, 187

Gratton, R. G., Carretta, E., \& Bragaglia, A. 2012, A\&ARv, 20, 50

Gustafsson, B., Edvardsson, B., Eriksson, K., et al. 2008, A\&A, 486, 951

Hendricks, B., Koch, A., Lanfranchi, G. A., et al. 2014, ApJ, 785, 102

Jönsson, H., Ryde, N., Nissen, P. E., et al. 2011, A\&A, 530, A144

Kaeufl, H.-U., Ballester, P., Biereichel, P., et al. 2004, in Ground-based Instrumentation for Astronomy, eds. A. F. M. Moorwood, \& M. Iye, SPIE Conf. Ser., 5492, 1218

Koch, A., \& Caffau, E. 2011, A\&A, 534, A52

Kupka, F., Piskunov, N., Ryabchikova, T. A., Stempels, H. C., \& Weiss, W. W. 1999, A\&AS, 138, 119

Kupka, F. G., Ryabchikova, T. A., Piskunov, N. E., Stempels, H. C., \& Weiss, W. W. 2000, Balt. Astron., 9, 590

Kurucz, R. L. 2004, Robert L. Kurucz on-line database of observed and predicted atomic transitions, http: //kurucz . harvard.edu/atoms

Kurucz, R. L. 2007, Robert L. Kurucz on-line database of observed and predicted atomic transitions, http: //kurucz .harvard.edu/atoms

Kurucz, R. L., Furenlid, I., Brault, J., \& Testerman, L. 1984, Solar flux atlas from 296 to 1300 nm (Sunspot, New Mexico: National Solar Observatory)

Lebzelter, T., Seifahrt, A., Uttenthaler, S., et al. 2012, A\&A, 539, A109

Limongi, M., \& Chieffi, A. 2003, ApJ, 592, 404
Marino, A. F., Villanova, S., Piotto, G., et al. 2008, A\&A, 490, 625 Marino, A. F., Milone, A. P., Piotto, G., et al. 2009, A\&A, 505, 1099

Marino, A. F., Sneden, C., Kraft, R. P., et al. 2011, A\&A, 532, A8

Matrozis, E., Ryde, N., \& Dupree, A. K. 2013, A\&A, 559, A115

Matteucci, F., \& Brocato, E. 1990, ApJ, 365, 539

McWilliam, A. 1997, ARA\&A, 35, 503

McWilliam, A., Preston, G. W., Sneden, C., \& Searle, L. 1995, AJ, 109, 2757

McWilliam, A., Wallerstein, G., \& Mottini, M. 2013, ApJ, 778, 149

Mishenina, T. V., Bienaymé, O., Gorbaneva, T. I., et al. 2006, A\&A, 456, 1109

Mishenina, T. V., Gorbaneva, T. I., Bienaymé, O., et al. 2007, Astron. Rep., 51, 382

Monaco, L., Villanova, S., Bonifacio, P., et al. 2012, A\&A, 539, A157

Nissen, P. E., Chen, Y. Q., Asplund, M., \& Pettini, M. 2004, A\&A, 415, 993

Nissen, P. E., Akerman, C., Asplund, M., et al. 2007, A\&A, 469, 319

Savage, B. D., \& Sembach, K. R. 1996, ARA\&A, 34, 279

Sbordone, L., Limongi, M., Chieffi, A., et al. 2009, A\&A, 503, 121

Shetrone, M. D., Côté, P., \& Sargent, W. L. W. 2001, ApJ, 548, 592

Shetrone, M., Venn, K. A., Tolstoy, E., et al. 2003, AJ, 125, 684

Spite, M., Caffau, E., Andrievsky, S. M., et al. 2011, A\&A, 528, A9

Takeda, Y., \& Takada-Hidai, M. 2011, PASJ, 63, 537

Takeda, Y., \& Takada-Hidai, M. 2012, PASJ, 64, 42

Takeda, Y., Hashimoto, O., Taguchi, H., et al. 2005, PASJ, 57, 751

Tinsley, B. M. 1979, ApJ, 229, 1046

Tolstoy, E., Hill, V., \& Tosi, M. 2009, ARA\&A, 47, 371

Ueda, Y., Mitsuda, K., Murakami, H., \& Matsushita, K. 2005, ApJ, 620, 274

Valenti, J. A., \& Piskunov, N. 1996, A\&AS, 118, 595

Venn, K. A., Irwin, M., Shetrone, M. D., et al. 2004, AJ, 128, 1177

Zerne, R., Caiyan, L., Berzinsh, U., \& Svanberg, S. 1997, Phys. Scr, 56, 459 